Article

\title{
Scaling of Second-Order Structure Functions in Turbulent Premixed Flames in the Flamelet Combustion Regime
}

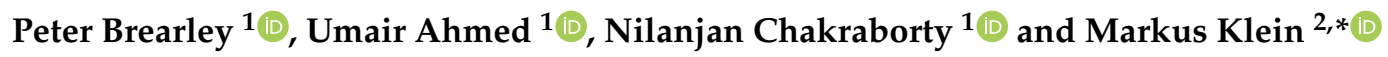 \\ 1 School of Engineering, Newcastle University, Newcastle-upon-Tyne NE1 7RU, UK; \\ p.brearley@newcastle.ac.uk (P.B.); umair.ahmed@newcastle.ac.uk (U.A.); \\ nilanjan.chakraborty@newcastle.ac.uk (N.C.) \\ 2 Bundeswehr University Munich, Department of Aerospace Engineering, LRT1, Werner-Heisenberg-Weg 39, \\ 85577 Neubiberg, Germany \\ * Correspondence: markus.klein@unibw.de
}

Received: 27 April 2020; Accepted: 28 May 2020; Published: 2 June 2020

\begin{abstract}
The second-order velocity structure function statistics have been analysed using a DNS database of statistically planar turbulent premixed flames subjected to unburned gas forcing. The flames considered here represent combustion for moderate values of Karlovitz number from the wrinkled flamelets to the thin reaction zones regimes of turbulent premixed combustion. It has been found that the second-order structure functions exhibit the theoretical asymptotic scalings in the dissipative and (relatively short) inertial ranges. However, the constant of proportionality for the theoretical asymptotic variation for the inertial range changes from one case to another, and this value also changes with structure function orientation. The variation of the structure functions for small length scale separation remains proportional to the square of the separation distance. However, the constant of proportionality for the limiting behaviour according to the separation distance square remains significantly different from the theoretical value obtained in isotropic turbulence. The disagreement increases with increasing turbulence intensity. It has been found that turbulent velocity fluctuations within the flame brush remain anisotropic for all cases considered here and this tendency strengthens towards the trailing edge of the flame brush. It indicates that the turbulence models derived based on the assumptions of homogeneous isotropic turbulence may not be fully valid for turbulent premixed flames.
\end{abstract}

Keywords: structure function; Kolmogorov scaling; premixed turbulent flames; direct numerical simulations

\section{Introduction}

Velocity structure functions (SFs) take a central role in turbulence theory [1-6], where they are mostly used to analyse the scale invariance of fully developed turbulence, characterized by a sequence of scaling exponents [7]. They have been measured up to the eighteenth order [4], primarily to establish the dependence on the order of the inertial range power-law exponent and to deduce information about the energy transfer distribution in the inertial range. Velocity (and scalar) SFs have been considered in a laboratory environment (see [8] for a summary of experimental results in various flow configurations) for a range of Reynolds numbers [8,9], but also for atmospheric shear flows [9] or in astrophysics [10]. Kolmogorov [1] developed his theory of locally homogeneous, isotropic turbulence by analyzing the second-order velocity SFs which forms the basis of many models used to close turbulence, thus second-order SFs play a fundamentally important role in understanding turbulence. Although SFs are 
widely used in the turbulence community to analyse experimental or numerical data, its application has been limited mostly to the analysis of non-reacting turbulent fluid flows.

The heat release rate due to combustion gives rise to flame normal acceleration and a significant magnitude of mostly positive dilatation rate due to thermal expansion. These effects lead to an augmentation of turbulence intensity within the turbulent premixed flame brush under some conditions, whereas turbulence decays across the flame brush under some other conditions. Furthermore, the flow field in turbulent premixed flames cannot be considered homogeneous and isotropic because of thermal expansion and preferential flow acceleration locally normal to the flame. The theoretical relations derived for homogeneous isotropic non-reacting turbulence are expected to be rendered invalid in premixed turbulent combustion. This suggests that the second-order SF behaviours in turbulent premixed flames can be significantly different from non-reacting flows.

SFs are commonly studied in conjunction with the correlation functions and their energy spectra [2]. Kolmogorov's famous $-5 / 3$ scaling law for the turbulent kinetic energy spectrum in the inertial range $\left(E(\kappa) \propto \kappa^{-5 / 3}\right.$ with $E(\kappa)$ and $\kappa$ being the turbulent kinetic energy spectrum and wavenumber, respectively) is equivalent to the $2 / 3$ scaling law for second-order structure functions (i.e., $\mathrm{SF} \propto r^{2 / 3}$ with $r$ being the separation distance) in physical space [1]. Since the turbulence is inherently inhomogeneous in turbulent premixed flames due to density change and preferred directionality associated with flame normal acceleration, the energy spectrum evaluation can be a challenging task. Although density-weighted correlation functions have been proposed [11], there are remaining questions regarding the differences in the turbulent kinetic energy spectrum between turbulent premixed flames and non-reacting flows [11]. As SFs depend only on velocity differences separated by spatial distances and do not rely upon Fourier transform to present the information in the spectral space, they have the potential to be more accessible than energy spectra to analyse turbulent flow structures in premixed turbulent combustion [12-15].

To date, the structure function behaviors in premixed flames have received relatively limited attention [12-15]. Sabelnikov et al. [12,14] analysed second-order SFs for weakly turbulent premixed flames in the corrugated flamelets regime [16] and analysed the SFs conditional upon different events (e.g., two points in reactants, two points in products, two points in the flame, one point in reactants and the other point in products). Recently, Brearley et al. [15] extended the analysis of Sabelnikov et al. [12,14] for flames belonging to the thin reaction zones regime [16] and revealed that the effects of heat release, which play key roles in the wrinkled flamelets regime, survive even for high Karlovitz number conditions in the thin reaction zones regime. In another study, Whitman et al. [13] analysed SFs conditioned on the flame normal and tangential directions for high turbulence intensities representative of high Karlovitz number, thin reaction zones regime combustion and indicated that Kolmogorov-type SF scaling laws might retain some validity in turbulent premixed flames. However, the scaling of the second-order velocity SFs within the flame brush belonging to different regimes of combustion are yet to be analysed in detail, and thus are investigated in this paper. Furthermore, the SF scaling constants for small and moderate separations associated with the inertial range according to Kolmogorov's theory [1] for homogeneous, isotropic turbulence have not yet been evaluated in turbulent premixed flames. Therefore, the differences in statistical behaviours of the second-order $\mathrm{SFs}$ in a turbulent premixed flame brush in comparison to the well-known asymptotic behaviours for non-reacting homogeneous isotropic turbulence are of fundamental importance from a modelling perspective, which motivates the current analysis.

A Direct Numerical Simulation (DNS) database $[15,17,18]$ of statistically planar flames ranging from the wrinkled flamelet to thin reaction zone regimes of premixed combustion has been considered for this analysis. The unburned gas turbulence is subject to isotropic forcing, which ensures desired values of both turbulence intensity and integral length scale upstream of the flame. 


\section{Mathematical Background and Numerical Implementation}

The second-order SFs are defined as [2,3]:

$$
D_{i j}(\mathbf{x}, \mathbf{r}, t)=\left\langle\left[u_{i}(\mathbf{x}+\mathbf{r}, t)-u_{i}(\mathbf{x}, t)\right]\left[u_{j}(\mathbf{x}+\mathbf{r}, t)-u_{j}(\mathbf{x}, t)\right]\right\rangle
$$

where $\mathbf{r}$ is the vector separating the points $\mathbf{x}$ and $\mathbf{x}+\mathbf{r}$ with magnitude $r=|\mathbf{r}|, t$ is time and $\langle\ldots\rangle$ refers to a suitable averaging operation. Equation (1) signifies the second moment of the velocity difference corresponding with eddies of size $r$. Numerous expressions can be derived from Eq. (1) for different flow configurations. For example, $D_{i j}(\mathbf{x}, \mathbf{r}, t)$ is independent of $\mathbf{x}$ in homogeneous turbulence, therefore $D_{i j}(\mathbf{r}, t)=2\left\langle u_{i}^{\prime} u_{j}^{\prime}\right\rangle-R_{i j}(\mathbf{r}, t)-R_{j i}(\mathbf{r}, t)$ where $u_{i}^{\prime}=u_{i}-\left\langle u_{i}\right\rangle$ is the velocity fluctuation in direction $i$ and $R_{i j}(\mathbf{r}, t)=\left\langle u_{i}^{\prime}(\mathbf{x}+\mathbf{r}, t) u_{j}^{\prime}(\mathbf{x}, t)\right\rangle$ is the two-point correlation tensor. $R_{i j}(\mathbf{r}, t)$ approaches zero for $r \gg \ell$, with $\ell$ being the integral length scale. Consequently, when $r \gg \ell$, $D_{i j}=2\left\langle u_{i}^{\prime} u_{j}^{\prime}\right\rangle$ in homogeneous turbulence which can be simplified further to $D_{i j}=2 u^{\prime 2} \delta_{i j}$ in isotropic turbulence, where $u^{\prime}=\sqrt{\left\langle u_{i}^{\prime} u_{i}^{\prime}\right\rangle / 3}$. Under homogeneous, incompressible turbulence, $D_{i j}(r, t)$ can be expressed completely in terms of the longitudinal SF $D_{L L}(r, t)=\left\langle\left[\mathbf{u}_{L}(\mathbf{x}+\mathbf{r}, t)-\mathbf{u}_{L}(\mathbf{x}, t)\right]^{2}\right\rangle$ as $D_{i j}(r, t)=D_{T T}(r, t) \delta_{i j}+\left[D_{L L}(r, t)-D_{T T}(r, t)\right] r_{i} r_{j} / r^{2}$, where the transverse SF $D_{T T}(r, t)=\left\langle\left[\mathbf{u}_{T}(\mathbf{x}+\right.\right.$ $\left.\left.\mathbf{r}, t)-\mathbf{u}_{T}(\mathbf{x}, t)\right]^{2}\right\rangle$ for homogeneous, incompressible turbulence is given by $D_{T T}(r, t)=D_{L L}(r, t)+$ $0.5 r\left[\partial D_{L L}(r, t) / \partial r\right]$, where $\mathbf{u}_{L}=(\mathbf{u} \cdot \mathbf{r}) \mathbf{r} / r^{2}$ is the component of the velocity in the direction of $\mathbf{r}$, and $\mathbf{u}_{T}=\mathbf{u}-\mathbf{u}_{L}$ is the velocity vector normal to $\mathbf{r}$. According to Kolmogorov's inertial range theory [1], one obtains $D_{L L}(\mathbf{r}, t)=C_{L}\langle\varepsilon r\rangle^{2 / 3}$ and $D_{T T}(\mathbf{r}, t)=4 D_{L L}(\mathbf{r}, t) / 3$ for $\eta<r<\ell$, with the universal constant $C_{L} \approx 2.0$ [2]. The kinetic energy dissipation rate is $\varepsilon=\mu\left(\partial u_{i} / \partial x_{j}\right)\left(\partial u_{i} / \partial x_{j}\right) / \rho$, with $\mu$ and $\rho$ being dynamic viscosity and density, respectively.

In premixed flames, the velocity field cannot be regarded as statistically homogeneous or isotropic and the assumption of incompressibility is invalid. Thus, it is not straightforward to have one-to-one correspondence between the correlation functions and second-order SFs in premixed flames. However, it is worthwhile to consider the relations based on non-reacting homogeneous isotropic turbulence so that it can be ascertained if $D_{L L}(\mathbf{r}, t) /\langle\varepsilon r\rangle^{2 / 3}$ and $D_{T T}(\mathbf{r}, t) /\langle\varepsilon r\rangle^{2 / 3}$ remain independent of $r$ for $\eta<r<\ell$ where $\eta=\left[\mu^{3} / \rho^{3}\langle\varepsilon\rangle\right]^{1 / 4}$ is the Kolmogorov length scale. Furthermore, it is important to evaluate if the ratios $D_{L L}(\mathbf{r}, t) /\langle\varepsilon r\rangle^{2 / 3}$ and $D_{T T}(\mathbf{r}, t) /\langle\varepsilon r\rangle^{2 / 3}$ are equal to $C_{L}$ and $4 C_{L} / 3$ according to Kolmogorov's theory [1] in turbulent reacting flows. For small separation distances (i.e., $r<\eta)$, one obtains $D_{i j} \propto\left(\partial u_{i} / \partial x_{k}\right)\left(\partial u_{j} / \partial x_{l}\right) r_{k} r_{l}$, which implies that $D_{L L}=C_{L}^{\prime}[\rho\langle\varepsilon\rangle / \mu] r^{2}$ and $D_{T T}=2 C_{L}^{\prime}[\rho\langle\varepsilon\rangle / \mu] r^{2}$ with $C_{L}^{\prime}=1 / 15$ for homogeneous isotropic turbulence [1,2].

The simulations comprising the current DNS database have been carried out using the well-known DNS code SENGA+ [19]. The simulation configuration consists of inlet and outlet boundaries in the direction of mean flame propagation, with the transverse boundaries being periodic. A tenth-order finite difference scheme has been used to calculate the spatial derivatives for the internal grid points. The scheme gradually reduces to second-order and one-sided at the non-periodic boundaries. An explicit, third-order Runge-Kutta scheme has been used for time advancement. The mean inlet velocity $U_{\text {mean }}$ has been gradually adjusted to match the turbulent flame speed for each case so the flame remains statistically stationary within the computational domain. The turbulent flame speed values are provided elsewhere [17] for this database and thus are not repeated here. The outflow boundary is assumed to be partially non-reflecting. Table 1 shows, from left to right, the root-mean-square turbulent velocity fluctuation in the unburned mixture normalised by the unperturbed laminar flame speed $u^{\prime} / S_{L}$, the integral length scale normalised by thermal flame thickness $\ell / \delta_{t h}$, the Damköhler number $D a=\ell S_{L} / u^{\prime} \delta_{t h}$, the Karlovitz number $K a=\left(u^{\prime} / S_{L}\right)^{3 / 2}\left(\ell / \delta_{t h}\right)^{-1 / 2}$, the heat release parameter $\tau=\left(T_{a d}-T_{0}\right) / T_{0}$, the physical size of the domain in terms of the flame thickness, the equidistant Cartesian grid used for discretization and finally, the corresponding regime of premixed combustion. In these equations, $\delta_{t h}=\left(T_{a d}-T_{0}\right) / \max |\nabla T|_{L}$ is the thermal flame thickness with $T_{a d}, T_{0}$ and $T$ being the adiabatic flame temperature, the unburned 
gas temperature and dimensional temperature respectively. The Cartesian grid provides at least 10 grid points within $\delta_{t h}$ and 1.5 grid points within the Kolmogorov length scale $\eta$ for all cases. The turbulence intensity $u^{\prime} / S_{L}$ and integral length scale $\ell / \delta_{t h}$ are maintained upstream of the flame using a modified bandwidth filtered physical space forcing method [20]. The cases considered in this analysis range from the wrinkled flamelet regime to the thin reaction zones regime, as shown in the Borghi-Peters diagram in Figure 1 [16]. In the present analysis, the chemical reaction is simplified by a single-step Arrhenius type reaction for the purpose of computational economy. As the analysis focuses on the velocity statistics, the simplification related to the chemical reaction is not expected to play a major role. This follows from the fact that the fluid velocity is affected by thermal expansion arising from heat release, and not by the intermediate steps of a chemical reaction. For example, the enstrophy transport characteristics obtained from simplified chemistry DNS [21,22] of turbulent premixed flames have been found to be qualitatively consistent with detailed chemistry results $[23,24]$. Thus, the findings regarding the SF statistics are likely to be qualitatively valid in the presence of detailed chemistry and transport. The Lewis number of all the species is taken to be unity and the specific heat of all the species is considered to be identical for the purpose of simplicity. These assumptions do not alter the qualitative nature of the heat release characteristics in turbulent premixed flames $[25,26]$ and the SF statistics are unlikely to be affected by these assumptions. The reaction progress variable is defined using the suitably normalised reactant mass fraction $Y_{R}$ by $c=\left(Y_{R 0}-Y_{R}\right) /\left(Y_{R 0}-Y_{R \infty}\right)$, where the subscripts 0 and $\infty$ refer to the quantity in the unburned and burned mixture, respectively. The simulations were continued until the turbulent kinetic energy $k$, integral length scale $\ell$, turbulent flame speed $S_{T}$ and flame surface area $A_{T}$ reach statistically stationary values. This duration was found to be longer than the throughpass time (i.e., $t_{\operatorname{sim}}>L_{x} / U_{\text {mean }}$ ) and 10 eddy turn over times (i.e., $\left.t_{\text {sim }}>10 \ell / u^{\prime}\right)$ for each case. The contours of $c$ for the DNS database considered have been provided elsewhere $[17,18]$ and are not repeated here.

Table 1. The properties of the DNS database considered in this analysis.

\begin{tabular}{ccccccccc}
\hline Case & $\boldsymbol{u}^{\prime} / S_{\boldsymbol{L}}$ & $\boldsymbol{\ell} / \boldsymbol{\delta}_{\boldsymbol{t h}}$ & $\mathbf{D a}$ & $\boldsymbol{K a}$ & $\boldsymbol{\tau}$ & Domain & Grid & Regime \\
\hline A & 1.0 & 3.0 & 3.00 & 0.577 & 4.5 & $79.5 \delta_{t h} \times\left(39.8 \delta_{t h}\right)^{2}$ & $800 \times 400^{2}$ & wrinkled flamelets \\
B & 2.5 & 3.0 & 1.20 & 2.28 & 4.5 & $79.5 \delta_{t h} \times\left(39.8 \delta_{t h}\right)^{2}$ & $800 \times 400^{2}$ & corrugated flamelets \\
C & 5.0 & 3.0 & 0.600 & 6.45 & 4.5 & $79.5 \delta_{t h} \times\left(39.8 \delta_{t h}\right)^{2}$ & $800 \times 400^{2}$ & thin reaction zones \\
D & 7.5 & 3.0 & 0.400 & 11.9 & 4.5 & $79.5 \delta_{t h} \times\left(39.8 \delta_{t h}\right)^{2}$ & $800 \times 400^{2}$ & thin reaction zones \\
E & 10 & 3.0 & 0.300 & 18.3 & 4.5 & $79.5 \delta_{t h} \times\left(39.8 \delta_{t h}\right)^{2}$ & $800 \times 400^{2}$ & thin reaction zones \\
\hline
\end{tabular}

In the case of statistically planar flames, the mean direction of propagation is parallel with the $x_{1}$-direction (right to left). This results in the $x_{2}-x_{3}$ plane at a given $x_{1}$ location to be statistically homogeneous. Thus, the analysis of the SFs has been limited to two sets of two points $\mathbf{x}_{A}=$ $\left\{x_{A B}, y_{A}, z_{A}\right\}$ and $\mathbf{x}_{B}=\left\{x_{A B}, y_{A}+r_{y}, z_{A}+r_{z}\right\}$. These points are located on the same transverse plane with coordinate $x_{A B}$ separated by the distance vector $\mathbf{r}=\left\{0, r_{y}, r_{z}\right\}$. The transverse SF $D_{11, T}(r)$ is calculated by considering $\left(u_{B, 1}-u_{A, 1}\right)^{2}$ and for two set of points given by $\mathbf{x}_{A}=\{x, y, z\}$; $\mathbf{x}_{B}=\{x, y+r, z\}$ and by $\mathbf{x}_{A}=\{x, y, z\} ; \mathbf{x}_{B}=\{x, y, z+r\}$. The transverse SFs $D_{22, T}(r)$ and $D_{33, T}(r)$ are evaluated by considering $\left(u_{B, 2}-u_{A, 2}\right)^{2}$ and $\left(u_{B^{\prime}, 3}-u_{A^{\prime}, 3}\right)^{2}$ respectively based on points $\mathbf{x}_{A}=\{x, y, z\} ; \mathbf{x}_{B}=\{x, y, z+r\}$ and $\mathbf{x}_{A^{\prime}}=\{x, y, z\} ; \mathbf{x}_{B \prime}=\{x, y+r, z\}$, respectively. Here, the notation $u_{B, 1}$ signifies the velocity component $u_{1}$ at point $\mathbf{x}_{B}$. Finally, the longitudinal SFs $D_{22, L}(r)$ and $D_{33, L}(r)$ are calculated by evaluating $\left(u_{B, 2}-u_{A, 2}\right)^{2}$ and $\left(u_{B^{\prime}, 3}-u_{A^{\prime}, 3}\right)^{2}$ respectively based on points $\mathbf{x}_{A}=\{x, y, z\} ; \mathbf{x}_{B}=\{x, y+r, z\}$ and $\mathbf{x}_{A^{\prime}}=\{x, y, z\} ; \mathbf{x}_{B \prime}=\{x, y, z+r\}$. This analysis considers $D_{23, T}(r)=0.5\left[D_{22, T}(r)+D_{33, T}(r)\right]$ and $D_{23, L}(r)=0.5\left[D_{22, L}(r)+D_{33, L}(r)\right]$ to account for the small departure from isotropy in the homogeneous directions (not to be confused with the cross-SF based on $u_{2}$ and $\left.u_{3}\right)$. 


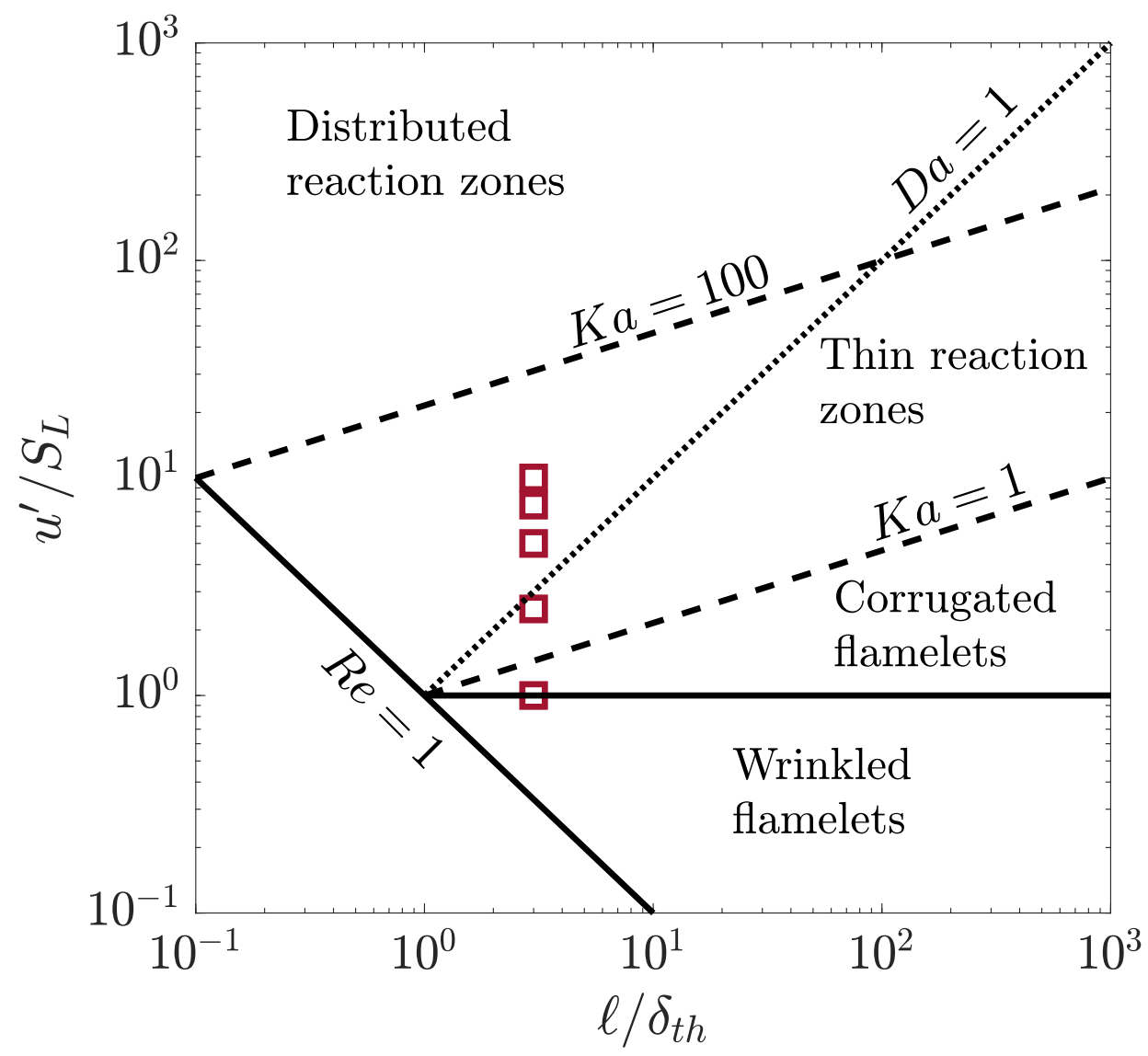

Figure 1. The cases considered in this analysis on a Borghi-Peters diagram.

\section{Results and Discussion}

The variations of $\left\{D_{11, T} /\left(\tau S_{L}\right)^{2}\right\}\left(r / \delta_{t h}\right)^{-2 / 3},\left\{D_{23, T} /\left(\tau S_{L}\right)^{2}\right\}\left(r / \delta_{t h}\right)^{-2 / 3}$ and $\left\{D_{23, L} /\left(\tau S_{L}\right)^{2}\right\}$ $\left(r / \delta_{t h}\right)^{-2 / 3}$ for all cases at the spatial location corresponding to the Reynolds averaged reaction progress variable $\bar{c}=0.5$ (where the overbar suggests a Reynolds averaging operation) are exemplarily shown in Figure $2 \mathrm{a}-\mathrm{c}$ respectively, where the SFs are conditionally averaged on bins of reaction progress variable $c$ for one of the points (i.e., $\mathbf{x}_{B}$ or $\mathbf{x}_{B^{\prime}}$ ) in question. This suggests that the samples associated with small values of $c$ in Figure 2 correspond to the events where $\mathbf{x}_{B}$ or $\mathbf{x}_{B^{\prime}}$ is in reactants (e.g., $c \leq 0.05$ ) and $\mathbf{x}_{A}$ or $\mathbf{x}_{A \prime}$ might be either in reactants or in products (e.g., $c \geq 0.95$ ) or in the flame (e.g., $0.05<c<0.95$ ). Similarly, large values of $c$ in Figure $2 \mathrm{a}-\mathrm{c}$ include samples from the events where $\mathbf{x}_{B}$ or $\mathbf{x}_{B^{\prime}}$ is in the products and $\mathbf{x}_{A}$ or $\mathbf{x}_{A^{\prime}}$ might be either in reactants or in products or within the flame.

The lines corresponding to the Kolmogorov scaling (i.e., $\left\{D_{11, T} /\left(\tau S_{L}\right)^{2}\right\}\left(r / \delta_{t h}\right)^{-2 / 3}=$ $\left(4 C_{L} / 3\right) \widetilde{\varepsilon}^{2 / 3} \delta_{t h}^{2 / 3} /\left(\tau S_{L}\right)^{2},\left\{D_{23, T} /\left(\tau S_{L}\right)^{2}\right\}\left(r / \delta_{t h}\right)^{-2 / 3}=\left(4 C_{L} / 3\right) \widetilde{\varepsilon}^{2 / 3} \delta_{t h}^{2 / 3} /\left(\tau S_{L}\right)^{2}$ and $\left\{D_{23, L} /\left(\tau S_{L}\right)^{2}\right\}$ $\left(r / \delta_{t h}\right)^{-2 / 3}=C_{L} \widetilde{\varepsilon}^{2 / 3} \delta_{t h}^{2 / 3} /\left(\tau S_{L}\right)^{2}$ and the limiting condition for small separation distances (i.e., $\left\{D_{11, T} /\left(\tau S_{L}\right)^{2}\right\}\left(r / \delta_{t h}\right)^{-2 / 3}=\left(2 C_{L}^{\prime}\right) \bar{\rho} \widetilde{\varepsilon} \delta_{t h}^{2 / 3} r^{4 / 3} / \mu\left(\tau S_{L}\right)^{2}, \quad\left\{D_{23, T} /\left(\tau S_{L}\right)^{2}\right\}\left(r / \delta_{t h}\right)^{-2 / 3}=$ $\left(2 C_{L}^{\prime}\right) \bar{\rho} \widetilde{\varepsilon} \delta_{t h}^{2 / 3} r^{4 / 3} / \mu\left(\tau S_{L}\right)^{2}$ and $\left\{D_{23, L} /\left(\tau S_{L}\right)^{2}\right\}\left(r / \delta_{t h}\right)^{-2 / 3}=C_{L}^{\prime} \bar{\rho} \widetilde{\varepsilon} \delta_{t h}^{2 / 3} r^{4 / 3} / \mu\left(\tau S_{L}\right)^{2}$ are shown by black dash and grey dash-dot lines, respectively. The qualitative behaviours of the SFs for other $\bar{c}$ values remain similar and thus are not shown for the sake of brevity. 


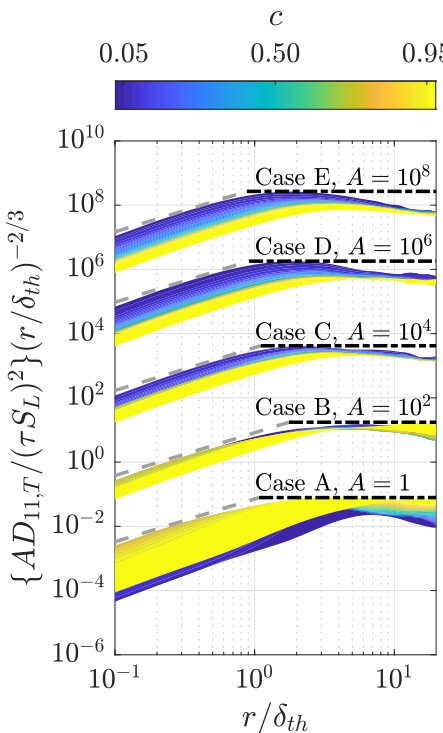

(a)

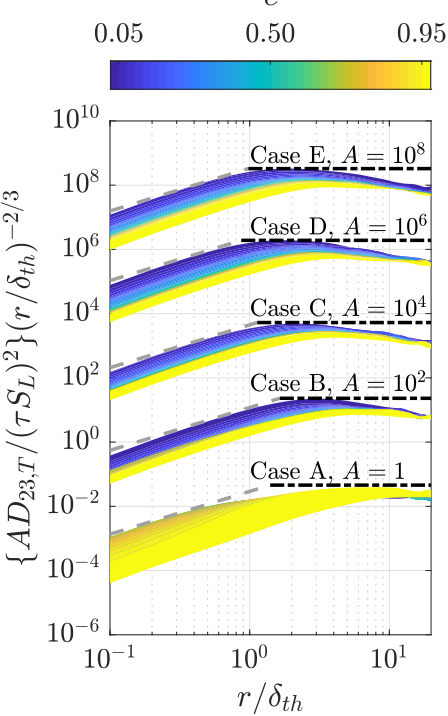

(b)

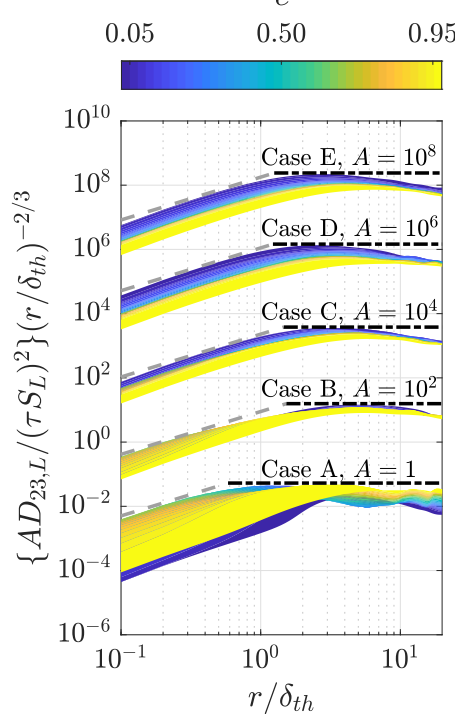

(c)

Figure 2. Variations of $\left\{A D_{11, T} /\left(\tau S_{L}\right)^{2}\right\}\left(r / \delta_{t h}\right)^{-2 / 3}, \quad\left\{A D_{23, T} /\left(\tau S_{L}\right)^{2}\right\}\left(r / \delta_{t h}\right)^{-2 / 3}$ and $\left\{A D_{23, L} /\left(\tau S_{L}\right)^{2}\right\}\left(r / \delta_{t h}\right)^{-2 / 3}$ (a-c) with $r / \delta_{t h}$ for cases A-E (from bottom to top) at $\bar{c}=0.5$ where $A=1,10^{2}, 10^{4}, 10^{6}$ and $10^{8}$ for cases A-E respectively. The analytic $r^{2}$ scaling and the Kolmogorov scaling $r^{2 / 3}$ are shown by grey dashed lines and black dash-dot lines respectively and the constants of proportionality used for these lines are shown in Figure 5. The colour bar indicates the values of $c$ for one of the points (i.e., $\mathbf{x}_{B}$ or $\mathbf{x}_{B^{\prime}}$ ) in question for the given value of $\bar{c}$. The coefficient $A$ is introduced for the convenience of presentation.

Figure 2 shows that the peak value of the SFs $\left\{D_{i j} /\left(\tau S_{L}\right)^{2}\right\}\left(r / \delta_{t h}\right)^{-2 / 3}$ is obtained around $r / \delta_{t h} \approx 2.0-4.0$ for all cases considered here. As expected, $\left\{D_{11, T} /\left(\tau S_{L}\right)^{2}\right\}\left(r / \delta_{t h}\right)^{-2 / 3}$, $\left\{D_{23, T} /\left(\tau S_{L}\right)^{2}\right\}\left(r / \delta_{t h}\right)^{-2 / 3}$ and $\left\{D_{23, L} /\left(\tau S_{L}\right)^{2}\right\}\left(r / \delta_{t h}\right)^{-2 / 3}$ all deviate from the Kolmogorov scaling, indicated by a plateau, for separation distances $r / \delta_{t h}>\ell / \delta_{t h}$, whereas for small separation distances these SFs exhibit reasonable agreement with a $D_{i j} \propto r^{2}$ scaling. The departure of the structure functions from the Kolmogorov scaling for the inertial range is not clearly visible in the previous results by Whitman et al. [13] due to a limited range of $r / \delta_{t h}$ values. Note that Whitman et al. [13] considered the SF behaviours in the local normal and tangential directions of flamelets, whereas this analysis focuses on the SFs in the normal and tangential directions of the mean flame brush. It has been demonstrated elsewhere $[23,27]$ that the relative strength of thermal expansion due to chemical heat release diminishes with increasing Karlovitz number, especially for high Karlovitz number within the thin reaction zones and the distributed reaction zones regimes of turbulent premixed combustion. Furthermore, turbulence forcing was used for the whole of the domain in Ref. [13], whereas in this analysis the forcing term is proportional to $(1-c)$ [20] to allow for the evolution of turbulence across the flame without the interference of numerical forcing. Despite these differences, the SFs, in particular, for cases C-E are in good agreement with the results presented in [13].

Note that substantial departures from the Kolmogorov scaling have been found previously even for non-reacting flows due to internal intermittency especially for low turbulent Reynolds numbers [5,28-30]. In order to better understand the observations made from Figure 2, the variations of Favre-averaged turbulent kinetic energy $\widetilde{k}=\overline{\rho u_{i}^{\prime \prime} u_{i}^{\prime \prime}} / 2 \bar{\rho}$ normalised by its value at $\bar{c}=0.001$ (representing the leading edge of the flame brush) are shown for cases $\mathrm{A}-\mathrm{E}$ as functions of Reynolds-averaged reaction progress variable $\bar{c}$ in Figure 3 . The values of $\widetilde{k}_{\bar{c}=0.001} / S_{L}^{2}$ are provided in Table 2 for the purpose of quantitative comparison between the different cases. Figure 3 shows that $\widetilde{k}$ increases significantly from the leading edge and decays only close to the trailing edge of the flame brush in case $\mathrm{A}$, whereas the augmentation of $\widetilde{k}$ from the leading edge of the flame brush in cases $B$ and $C$ is followed by a decay before another increasing trend is observed in the region of 
the flame brush where the effects of chemical reaction and heat release are significant (i.e., where the probability of finding $0.5 \leq c \leq 0.9$ remains significant) before turbulence decays towards the product side of the flame brush. The augmentation of $\widetilde{k}$ in the region of the flame brush where the effects of heat release are significant weakens progressively from case $B$ to case $C$. The turbulent kinetic energy decays monotonically from the leading edge to the trailing edge of the flame brush in cases $\mathrm{D}$ and E. The observations from Figure 3 indicate that turbulence is sustained for the major part of the flame brush and it gets locally augmented within some parts of the flame brush in cases A, B and C in decreasing order, whereas turbulence decays across the flame brush in cases D and E. Moreover, Figure 2a-c reveal that the mean values of the SFs attain high levels for high values of $c$ for cases $A$ and $\mathrm{B}$, whereas cases C-E show the greatest value is attained at low values of $c$. These results agree with those by Whitman et al. [13]. While the SFs seem to be ordered according to the value of $c$ for cases C-E, some SFs with different $c$ value cross each other for case A. This general behaviour can be deduced from the longitudinal SF $D_{11, L}$ of a laminar 1D flame, as shown in Figure 4, and by noting that the local flame normal is not aligned with the mean direction of flame propagation in Figure 2a-c, such that for example $D_{11, T}$ contains samples of the longitudinal 1D SF. Figure 4 shows that for small separation distances, the magnitude of $D_{11, L}$ increases with increasing $c$ before attaining a maximum around $c \approx 0.5$. Moreover, some of the SFs starting from a higher value of $c$ intercept the SFs starting from a lower value of $c$. Finally, it is noted that case A with a low $u^{\prime} / S_{L}$ belongs to the wrinkled flamelet regime and case B shows a behaviour in-between case A and cases C-E. For $r / \delta_{t h} \gg \ell / \delta_{t h}$, the ordering of all SF magnitudes for all cases is in agreement with the approximation $D_{i j}=2\left\langle u_{i}^{\prime} u_{j}^{\prime}\right\rangle$ [15] together with the increasing, respectively decreasing, trends of $\widetilde{k}$ as reported in Figure 3 . The inertial range plateau in cases A and B extends to higher values of $r / \delta_{t h}$, presumably caused by the relatively strong effects of heat release and the associated elongation of flow structures.

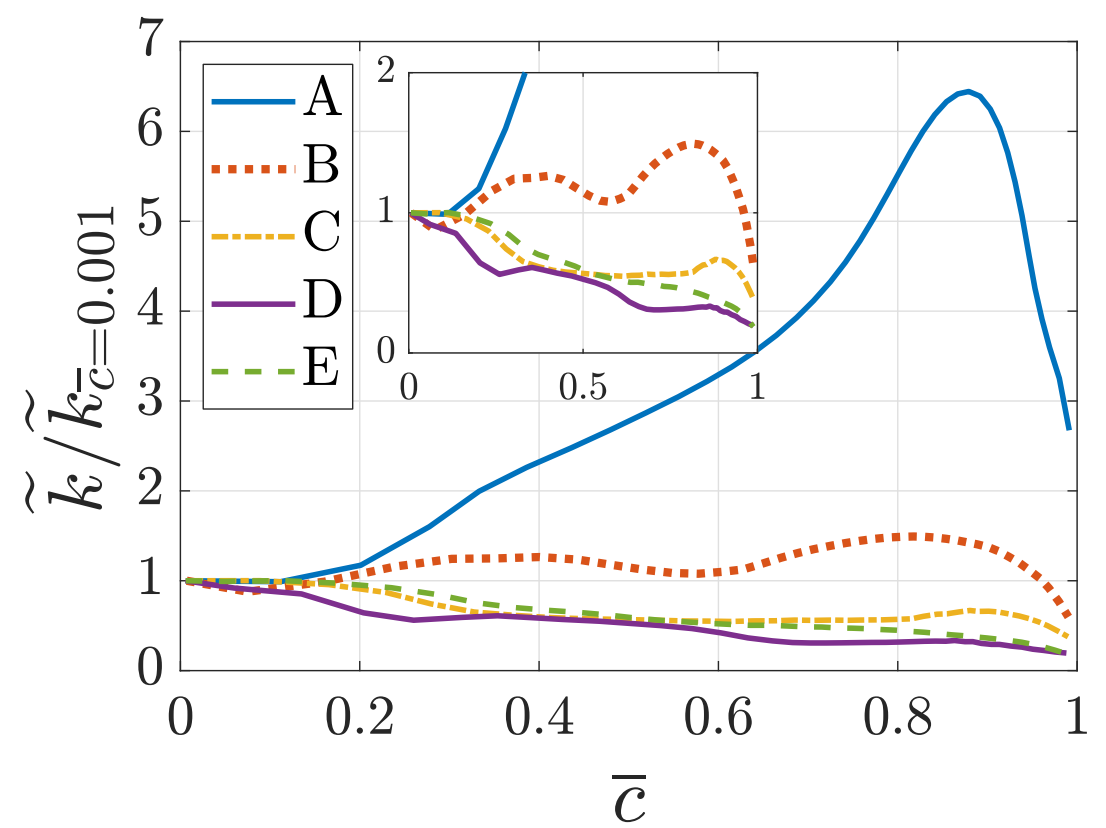

Figure 3. Variations of $\widetilde{k}$ normalised by its value at $\bar{c}=0.001$ with $\bar{c}$ for cases A-E.

Table 2. Values of $\widetilde{k}_{\bar{c}=0.001} / S_{L}^{2}$ and $\widetilde{\varepsilon}_{\bar{c}=0.001} \delta_{t h} / S_{L}^{3}$ for cases A-E.

\begin{tabular}{ccc}
\hline Case & $\widetilde{\boldsymbol{k}}_{\overline{\boldsymbol{c}}=\mathbf{0 . 0 0 1}} / S_{\mathbf{L}}^{2}$ & $\widetilde{\boldsymbol{\varepsilon}}_{\overline{\boldsymbol{c}}=\mathbf{0 . 0 0 1}} \delta_{t h} / S_{\mathbf{L}}^{3}$ \\
\hline A & 0.727 & 0.132 \\
B & 6.31 & 4.18 \\
C & 20.0 & 54.5 \\
D & 87.0 & 209 \\
E & 124 & 356 \\
\hline
\end{tabular}




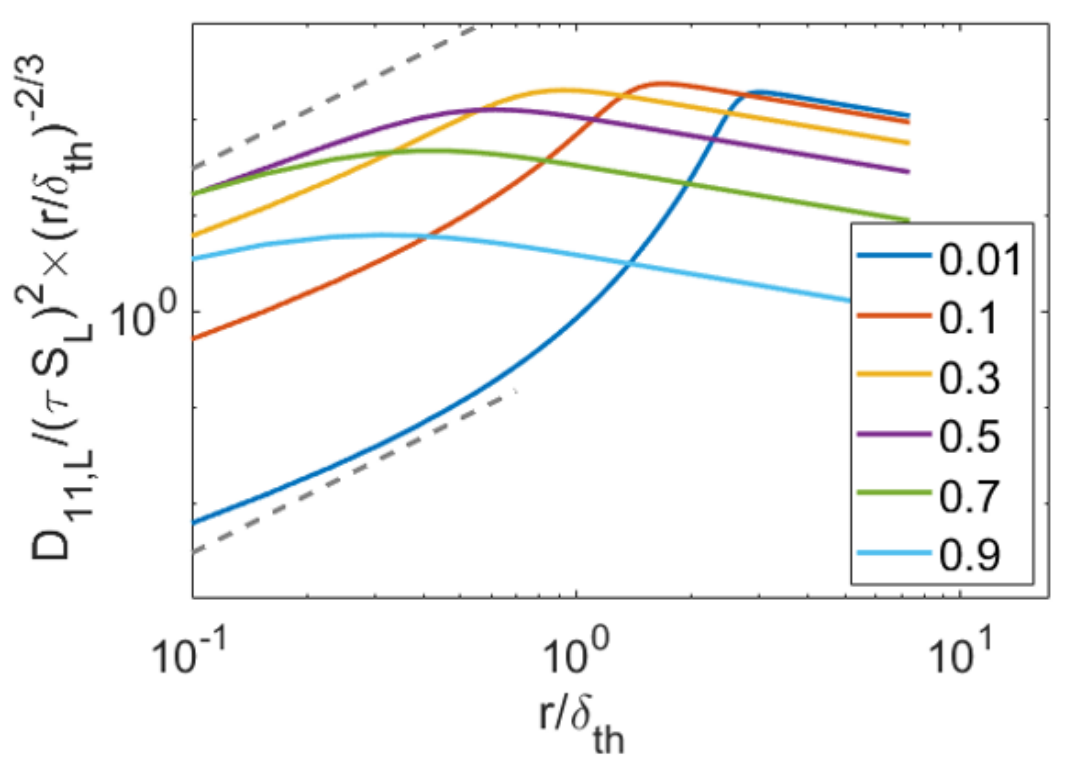

Figure 4. Variations $\left\{D_{11, L} /\left(\tau S_{L}\right)^{2}\right\}\left(r / \delta_{t h}\right)^{-2 / 3}$ with $r / \delta_{t h}$ for a laminar 1D flame for SFs starting from different $c$ values. The analytic $r^{2}$ scaling is shown by grey dashed lines.

The variation of the proportionality parameters (i.e., $C_{L}=\left\{3 D_{11, T} / 4\right\}(\widetilde{\varepsilon} r)^{-2 / 3}, C_{L}^{\prime}=$ $\left\{D_{11, T} \mu / 2 \bar{\rho} \widetilde{\varepsilon}\right\}(r)^{-2} ; C_{L}=\left\{3 D_{23, T} / 4\right\}(\widetilde{\varepsilon} r)^{-2 / 3}, C_{L}^{\prime}=\left\{D_{23, T} \mu / 2 \widetilde{\rho} \widetilde{\varepsilon}\right\}(r)^{-2}$ and $C_{L}=\left\{D_{23, L}\right\}(\widetilde{\varepsilon} r)^{-2 / 3}$, $\left.C_{L}^{\prime}=\left\{D_{23, L} \mu / \widetilde{\rho} \widetilde{\varepsilon}\right\}(r)^{-2}\right)$ corresponding to the dash and dash-dot lines showing the limiting behaviours in Figure 2 for cases A-E are shown in Figure 5, which indicates that both $C_{L}$ and $C_{L}^{\prime}$ values are different for $D_{11, T}, D_{23, T}$ and $D_{23, L}$, and these values change from one case to another. However, for all cases $C_{L}$ remains of the order of 1.5 (which is close to the theoretical value of 2.0), whereas $C_{L}^{\prime}$ remains significantly smaller than $1 / 15$ for all cases considered here and $C_{L}^{\prime}$ decreases from case A to case $\mathrm{E}$. The departures from Kolmogorov scaling can be explained in the following manner. The Kolmogorov scaling is applicable for homogeneous isotropic turbulence in the inertial range. However, the inertial range possibly is not fully observed for these cases because of the moderate values of turbulent Reynolds number and the isotropy is disturbed by the flow acceleration within the flame. In addition to that, the dissipation rate $\widetilde{\varepsilon}$ variation within these flames is significantly affected by the heat release, which can be substantiated from Figure 6 where the variations of $\widetilde{\varepsilon}$ normalised by its value at $\bar{c}=0.001$ representing the leading edge of the flame brush are shown for cases A-E as functions of $\bar{c}$. The values of $\widetilde{\mathcal{E}}_{\bar{c}=0.001} \delta_{t h} / S_{L}^{3}$ are also provided in Table 2 for the purpose of quantitative comparison. A comparison between Figures 3 and 6 reveals that the variation of $\widetilde{\mathcal{\varepsilon}}$ within the flame brush is qualitatively similar to that of $\widetilde{k}$. This suggests that the thermal expansion within the flame brush has a significant influence on $\widetilde{\varepsilon}$, which is absent in the classical turbulence theory by Kolmogrorov [1]. Furthermore, the nature of the underlying flow within the flame brush can be characterised with the help of the Lumley triangle [2]. The Lumley triangles for the limiting cases A and E are exemplarily shown in Figure 7 where $\xi$ and $\eta$ are given by

$$
6 \xi^{3}=b_{i j} b_{j k} b_{k i} ; \quad 6 \eta^{2}=b_{i j} b_{j i}
$$

where $b_{i j}=\widetilde{u_{i}^{\prime \prime} u_{j}^{\prime \prime}} / 2 \widetilde{k}-\delta_{i j} / 3$ is the normalised Reynolds stress anisotropy tensor. Figure 7 shows that the underlying turbulence within the flame brush is anisotropic for cases $\mathrm{A}$ and $\mathrm{E}$, with cases $\mathrm{B}, \mathrm{C}$ and $\mathrm{D}$ following the same trend. Though, the extent of anisotropy in the unburned gas side of the flame brush decreases with increasing $u^{\prime} / S_{L}$. However, turbulence becomes anisotropic for all cases within the flame brush, and this tendency strengthens with increasing $\bar{c}$ within the flame brush. This indicates that the limiting conditions based on homogeneous isotropic turbulence may not be applicable for the second-order SFs within the flame brush for moderate values of Ka. This also indicates that the assumption of isotropy is rendered invalid in the modelling for turbulent premixed 
flames for moderate values of Karlovitz number. This is consistent with recent findings in the context of the closures of sub-grid scalar flux [31] and stresses [18,32] in turbulent premixed combustion.

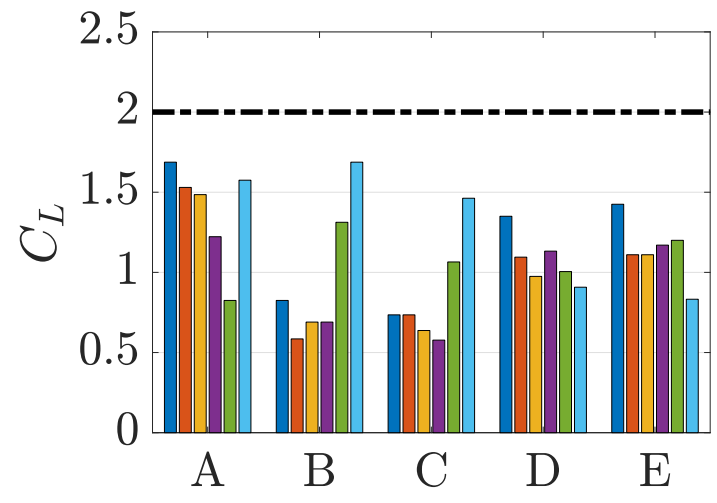

Case

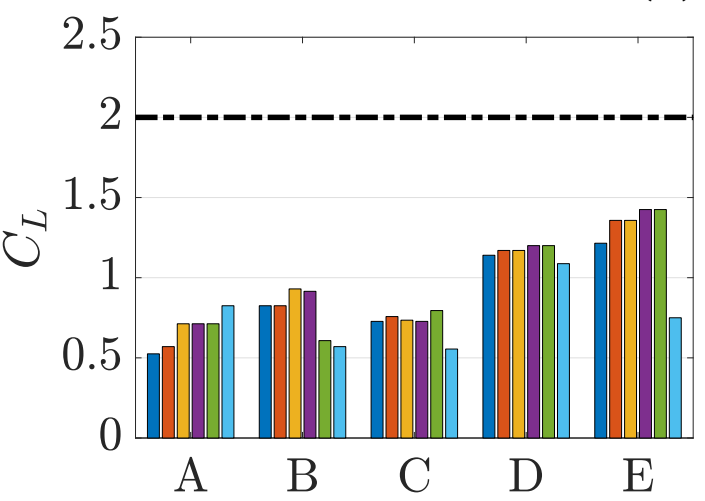

Case

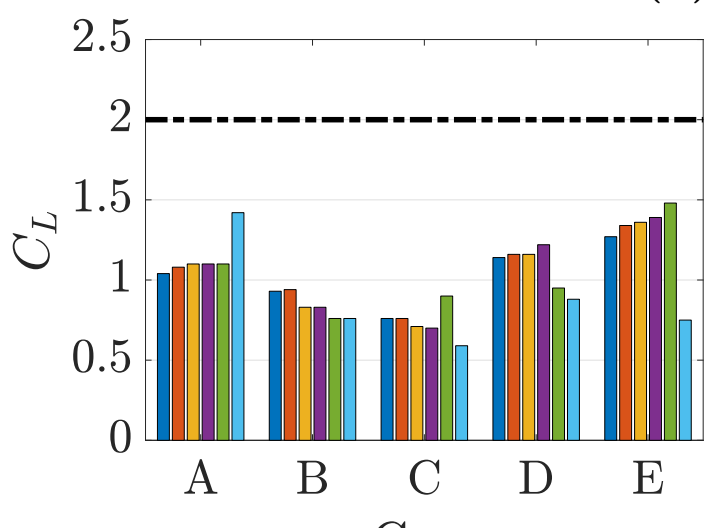

Case

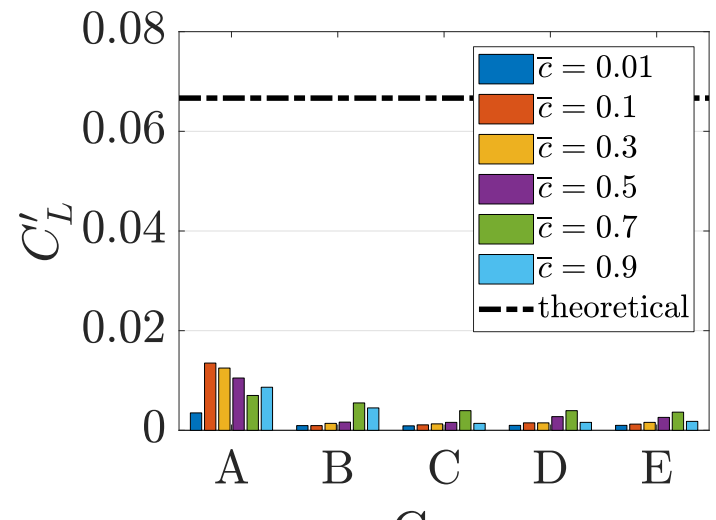

Case

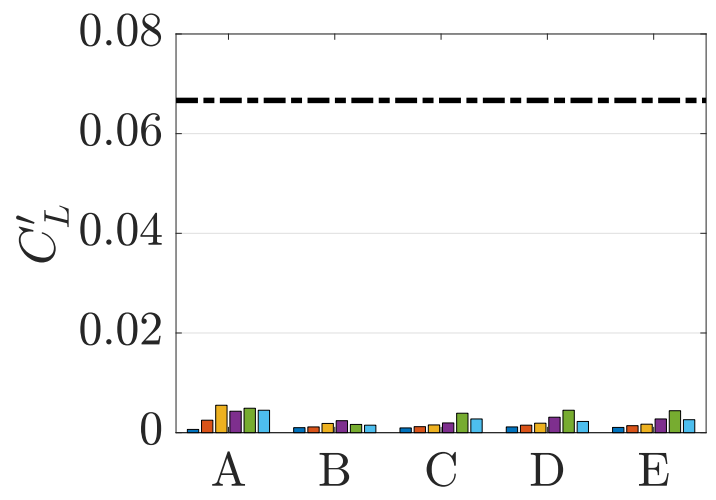

Case

(b) $D_{23, T}$

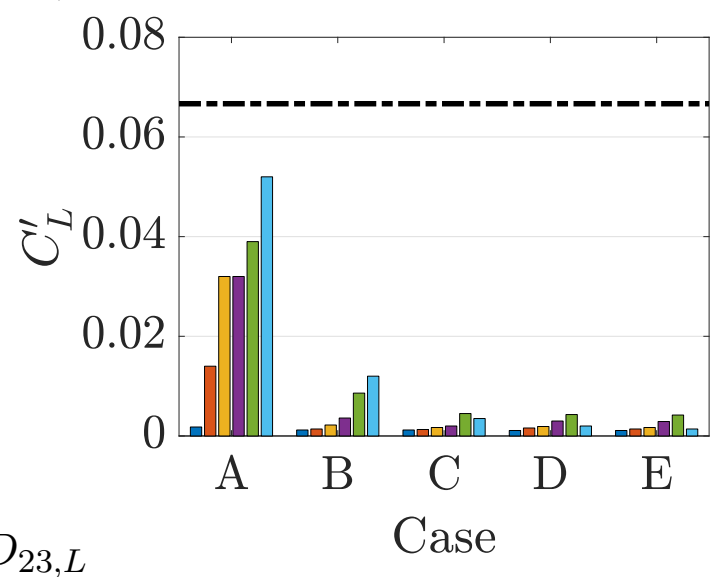

Figure 5. Variations of the proportionality parameters (i.e., $C_{L}=\left\{3 D_{11, T} / 4\right\}(\widetilde{\varepsilon} r)^{-2 / 3}, C_{L}^{\prime}=$ $\left\{D_{11, T} \mu / 2 \widetilde{\rho} \widetilde{\varepsilon}\right\}(r)^{-2} ; C_{L}=\left\{3 D_{23, T} / 4\right\}(\widetilde{\varepsilon} r)^{-2 / 3}, C_{L}^{\prime}=\left\{D_{23, T} \mu / 2 \widetilde{\rho} \widetilde{\varepsilon}\right\}(r)^{-2}$ and $C_{L}=\left\{D_{23, L}\right\}(\widetilde{\varepsilon} r)^{-2 / 3}$, $\left.C_{L}^{\prime}=\left\{D_{23, L} \mu / \widetilde{\rho} \widetilde{\varepsilon}\right\}(r)^{-2}\right)$ for cases A-E for (a) $D_{11, T}$, (b) $D_{23, T}$, (c) $D_{23, L}$, respectively. The horizontal lines show the asymptotic values based on Kolmogorov scaling (i.e., $C_{L}=2.0$ ) and homogeneous isotropic turbulence (i.e., $C_{L}^{\prime}=1 / 15$ ) assumption for $C_{L}$ and $C_{L}^{\prime}$, respectively. 


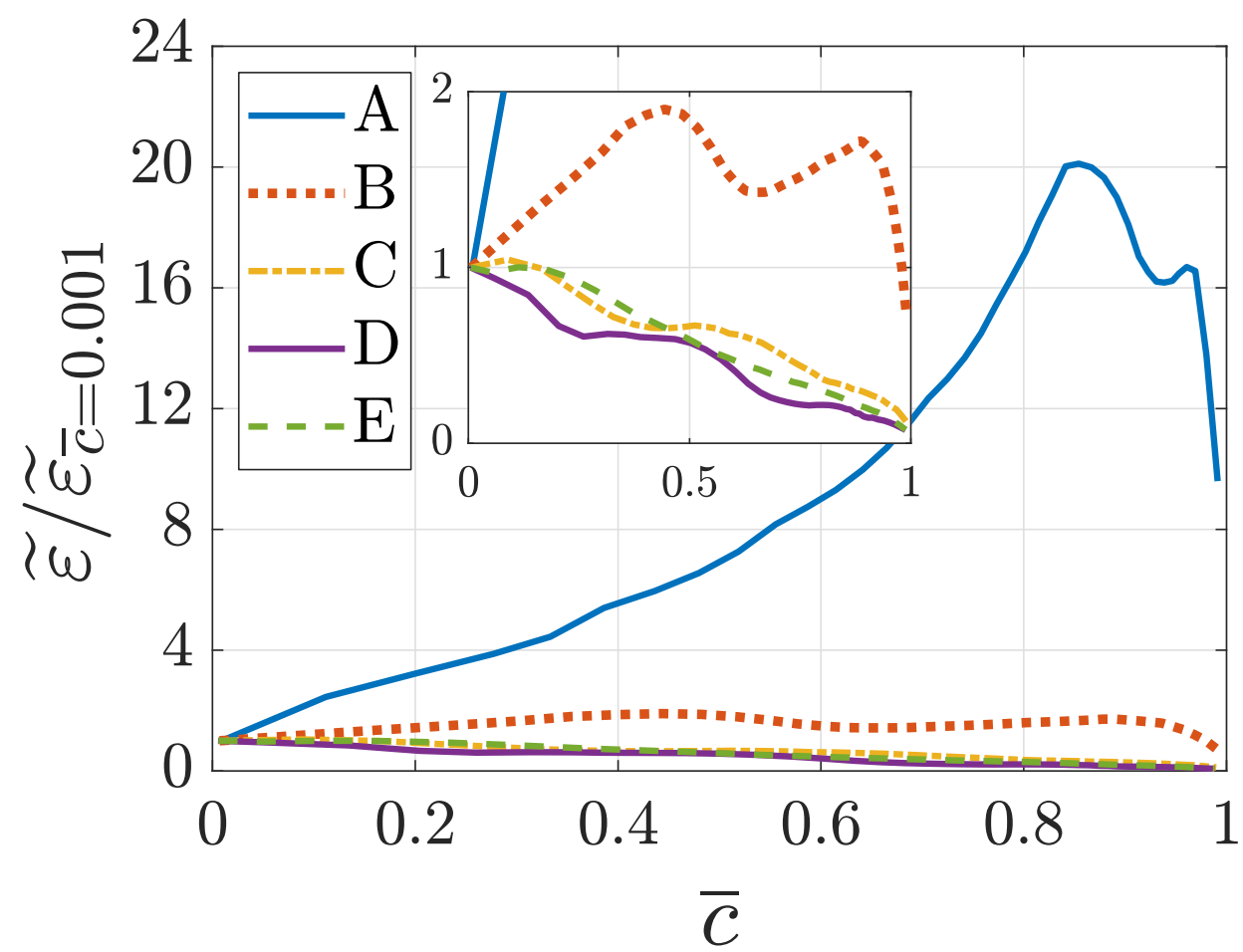

Figure 6. Variations of normalised $\widetilde{\varepsilon}$ normalised by its value at $\bar{c}=0.001$ with $\bar{c}$ for cases A-E.

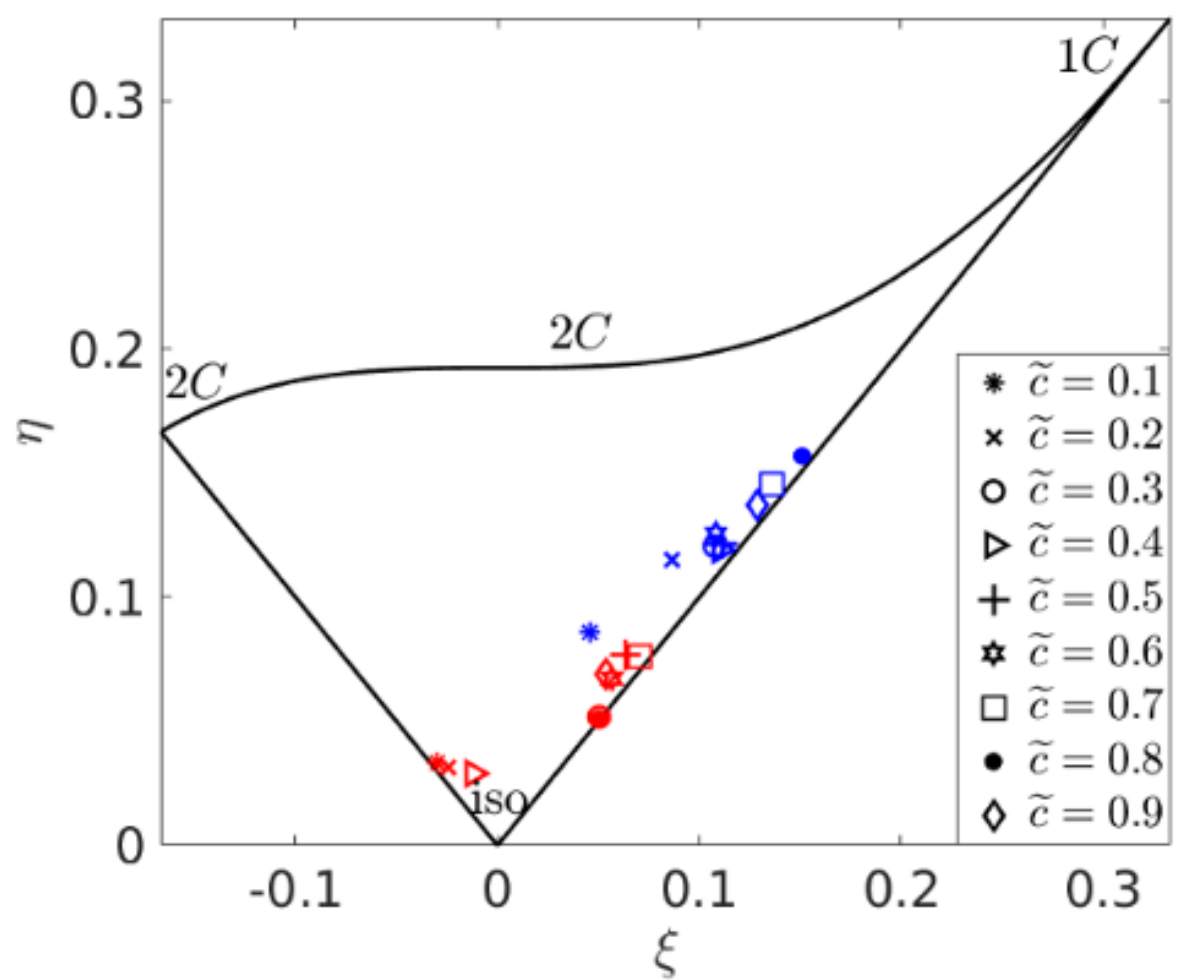

Figure 7. Lumley triangle on the plane of the invariants $\xi$ and $\eta$ of the Reynolds stress anisotropy tensor for case A (blue) and $\mathrm{E}$ (red) for different values of $\bar{c}$. $1 C, 2 C$ and iso are the one component limit, two component limit and isotropic respectively.

\section{Conclusions}

The statistical behaviours of second-order velocity SFs have been analysed based on a DNS database of statistically planar turbulent premixed flames. The simulation parameters have been 
chosen such that the cases considered here span from the wrinkled flamelets to the thin reaction zones regimes of turbulent premixed combustion. The second-order SFs for these flames show, especially for high values of turbulence, the theoretical scalings but considerable departures from the expected constants of proportionality. These departures of the constants are more prominent in the flames with higher values of $u^{\prime} / S_{L}$ among the cases considered here. Moreover, the constant of proportionality $C_{L}=D_{i j}\langle\widetilde{\varepsilon} r\rangle^{-2 / 3}$ for the theoretical asymptotic (i.e., $D_{i j} \propto\langle\widetilde{\varepsilon} r\rangle^{2 / 3}$ ) variation changes from one case to another although it has roughly the correct order of magnitude. For short separation distances, the second-order velocity SFs have been found to be proportional to $r^{2}$ (i.e. $D_{i j} \propto r^{2}$ ) but the constants of proportionality $C_{L}^{\prime}=\left\{D_{i j} \mu / \bar{\rho} \widetilde{\varepsilon}\right\}(r)^{-2}$ are significantly smaller than $1 / 15$, which is obtained based on the assumption of homogeneous isotropic turbulence. It has been demonstrated based on the Lumley triangle that the underlying turbulent flow within the flame brush is highly anisotropic for all cases within the flame brush and this tendency strengthens with increasing $\bar{c}$ within the flame brush. This indicates that the assumptions of homogeneous isotropic turbulence may not be accurate for turbulent combustion modelling for moderate values of Karlovitz number.

The present analysis has been carried out with simple chemistry and molecular transport. Although these assumptions are unlikely to alter the qualitative nature of the findings, further investigations in the presence of detailed chemistry and transport will be necessary for a more comprehensive understanding of the SF statistics in turbulent premixed flames.

Author Contributions: Conceptualization, P.B., U.A., N.C. and M.K.; methodology, P.B., U.A. and N.C.; formal analysis, P.B., U.A. and N.C.; writing - original draft preparation, N.C., P.B. and M.K.; writing-review and editing, U.A. and N.C.; supervision, N.C.; funding acquisition, N.C. All authors have read and agreed to the published version of the manuscript.

Funding: This research was funded by the Engineering and Physical Sciences Research Council, grant number $\mathrm{EP} / \mathrm{R} 029369 / 1$.

Acknowledgments: The authors are grateful to the Archer and Rocket HPC services for computational support

Conflicts of Interest: The authors declare no conflict of interest.

\section{References}

1. Kolmogorov, A.N. The local structure of turbulence in incompressible viscous fluid for very large Reynolds number. Dokl. Akad. Nauk SSSR 1941, 30, 299-303.

2. Pope, S.B. Turbulent Flows; Cambridge University Press: Cambridge, UK, 2000.

3. Frisch, U. The Legacy of A. N. Kolmogorov; Cambridge University Press: Cambridge, UK, 1995.

4. Anselmet, F.; Gagne, Y.; Hopfinger, E.J.; Antonia, R.A. High-order velocity structure functions in turbulent shear flows. J. Fluid Mech. 1984, 140, 63-89. [CrossRef]

5. Sreenivasan, K.R.; Antonia, R. The phenomenology of small scale turbulence. Annu. Rev. Fluid Mech. 1997, 29, 435-472. [CrossRef]

6. Lohse, D.; Xia, K.-Q. Small-Scale Properties of Turbulent Rayleigh-Bénard Convection. Annu. Rev. Fluid Mech. 2010, 42, 335-364. [CrossRef]

7. Huang, Y.X.; Schmitt; F.G.; Lu, Z.M.; Fougairolles, P.; Gagne, Y.; Liu, Y.L. Second-order structure function in fully developed turbulence. Phys. Rev. E 2010, 82, 026319. [CrossRef]

8. Arneodo, A.; Baudet, C.; Belin, F.; Benzi, R.; Castaing, B.; Chabaud, B.; Chavarria, R.; Ciliberto, S.; Camussi, R.; Chillà, F.; et al. Structure functions in turbulence, in various flow configurations, at Reynolds number between 30 and 5000, using extended self-similarity. Europhys. Lett. 1996, 34, 411-416. [CrossRef]

9. Antonia, R.A.; Satyaprakash, B.R.; Chambers, A.J. Reynolds number dependence of velocity structure functions in turbulent shear flows Phys. Fluids 1982, 25, 29-37.

10. Heyer, M.H.; Brunt, C.M. The Universality of Turbulence in Galactic Molecular Clouds. Astrophys. J. 2004, 615, L45-L48. [CrossRef]

11. Kolla, H.; Hawkes, E. R.; Kerstein, A.R.; Swaminathan, N; Chen, J.H. On velocity and reactive scalar spectra in turbulent premixed flames. J. Fluid Mech. 2014, 754, 456-487. [CrossRef] 
12. Sabelnikov, V.A.; Lipatnikov, A.N.; Nishiki, S.; Hasegawa, T. Application of conditioned structure functions to exploring influence of premixed combustion on two-point turbulence statistics. Proc. Combust. Inst. 2019, 37, 2433-2441. [CrossRef]

13. Whitman, S.H.R.; Towery, C.A.Z.; Poludnenko, A.Y.; Hamlington, P.E. Scaling and collapse of conditional velocity structure functions in turbulent premixed flames. Proc. Combust. Inst. 2019, 37, 2427-2435. [CrossRef]

14. Sabelnikov, V.A.; Lipatnikov, A.N.; Nishiki, S.; Hasegawa, T. Investigation of the influence of combustion-induced thermal expansion on two-point turbulence statistics using conditioned structure functions. J. Fluid. Mech. 2019, 867, 45-76. [CrossRef]

15. Brearley, P.; Ahmed, U.; Chakraborty, N.; Lipatnikov, A. N. Statistical behaviours of conditioned two-point second-order structure functions in turbulent premixed flames in different combustion regimes. Phys. Fluids 2019, 31, 115109. [CrossRef]

16. Peters, N. Turbulent Combustion; Cambridge University Press: Cambridge, UK, 2000.

17. Ahmed, U.; Chakraborty, N.; Klein, M. Insights into the bending effect in premixed turbulent combustion using the Flame Surface Density transport. Combust. Sci. Technol. 2019, 191, 898-920. [CrossRef]

18. Ahmed, U.; Chakraborty, N.; Klein, M. On the stress-strain alignment in premixed turbulent flames. Sci. Rep. 2019, 9, 5092. [CrossRef] [PubMed]

19. Jenkins, K. W.; Cant, R. S. Direct numerical simulation of turbulent flame kernels. In Recent Advances in DNS and LES: Proceedings of the Second AFOSR Conference, Rutgers, New Brunswick, NJ, USA, 7-9 June 1999; Springer: Berlin/Heidelberg, Germany, 1999; pp. 191-202.

20. Klein, M.; Chakraborty, N.; Ketterl, S. A comparison of strategies for direct numerical simulation of turbulence chemistry interaction in generic planar turbulent premixed flames. Flow Turbul. Combust. 2017, 99, 955-971. [CrossRef]

21. Chakraborty, N.; Konstantinou, I.; Lipatnikov, A. Effects of Lewis number on vorticity and enstrophy transport in turbulent premixed flames. Phys. Fluids 2016, 28, 015109. [CrossRef]

22. Doan, N.A.K.; Swaminathan, N.; Chakraborty, N. Multiscale analysis of turbulence-flame interaction in premixed flames. Proc. Combust. Inst. 2017, 36, 1929-1935. [CrossRef]

23. Papapostolou, V.; Wacks, D.H.; Klein, M.; Chakraborty, N.; Im, H.G. Enstrophy transport conditional on local flow topologies in different regimes of premixed turbulent combustion. Sci. Rep. 2017, 7, 11545. [CrossRef]

24. Ahmed, U.; Doan, N.A.K.; Lai., J.; Klein, M.; Chakraborty, N.; Swaminathan, N. Multiscale analysis of head-on quenching premixed turbulent flames. Phys. Fluids 2018, 30, 105102. [CrossRef]

25. Poinsot, T.; Echekki, T.; Mungal, M.G. A study of the laminar flame tip and implications for premixed turbulent combustion. Combust. Sci. Technol. 1992, 81, 45-73. [CrossRef]

26. Louch, D.S.; Bray, K.N.C. Vorticity in unsteady premixed flames: Vortex pair-premixed flame interactions under imposed body forces and various degrees of heat release and laminar flame thickness. Combust. Flame 2001, 125, 1279-1309. [CrossRef]

27. Wacks, D.H.; Chakraborty, N.; Klein, M.; Arias, P.G; Im, H.G. Flow topologies in different regimes of premixed turbulent combustion: A direct numerical simulation analysis. Phys. Rev. Fluids 2016, 1, 083401. [CrossRef]

28. Kolmogorov, A.N. A refinement of previous hypotheses concerning the local structure of turbulence in a viscous incompressible fluid at high Reynolds number. J. Fluid Mech. 1962, 13, 82-85. [CrossRef]

29. Cao, N.; Chen, S.; Sreenivasan, K. R. Scaling of low-order structure functions in homogeneous turbulence. Phys. Rev. Lett. 1996, 77, 3799-3802. [CrossRef]

30. Antonia, R.A.; Djenidi, L.; Danaila, L.; Tang, S.L. Small scale turbulence and the finite Reynolds number effect. Phys. Fluids 2017, 29, 020715. [CrossRef]

31. Klein, M.; Kasten, C.; Chakraborty, N.; Gao, Y.; A-priori Direct Numerical Simulation assessment of sub-grid scale stress tensor closures for turbulent premixed combustion. Comp. Fluids 2015, 122, 1-11. [CrossRef]

32. Klein, M.; Kasten, C.; Chakraborty, N.; Mukhadivev, N.; Im, H.G. Turbulent scalar fluxes in Hydrogen-Air premixed flames at low and high Karlovitz numbers. Combust. Theor. Model. 2018, 22, 1-16. [CrossRef]

(C) 2020 by the authors. Licensee MDPI, Basel, Switzerland. This article is an open access article distributed under the terms and conditions of the Creative Commons Attribution (CC BY) license (http:/ / creativecommons.org/licenses/by/4.0/). 